\title{
TENDÊNCIAS DAS PESQUISAS DE ENFERMAGEM PSIQUIÁTRICA E SAÚDE MENTAL NO PERÍODO DE 1998 A 2008
}

\author{
Belisa Vieira da Silveira ${ }^{1}$, Amanda Nathale Soares², Amanda Márcia dos Santos Reinaldo²
}

RESUMO: Objetiva-se analisar a produção científica nos Encontros de Pesquisadores em Saúde Mental e Encontro de Especialistas em Enfermagem Psiquiátrica, em relação às mudanças ocorridas e às políticas de Saúde Mental no país, no período de 1998 a 2008. Categorizaram-se os resumos em 11 núcleos temáticos, apresentados em uma tabela demonstrativa, sendo a Reforma Psiquiátrica e o Ensino em Saúde Mental os principais eixos de produção científica. A situação ideológica e social de cada ano relaciona-se às produções científicas, indicando que o meio acadêmico e a realidade assistencial caminham conjuntamente.

PALAVRAS-CHAVE: Saúde mental; Enfermagem psiquiátrica; Pesquisa.

\section{TRENDS OF PSYCHIATRIC NURSING AND MENTAL HEALTH RESEARCHES FROM 1998 TO 2008}

\begin{abstract}
This article aims to analyze the scientific production in the Meetings of Mental Health Researchers and Meeting of Experts in Psychiatric Nursing regarding the changes and policies in Mental Health from 1998 to 2008. Summaries are categorized into 11 thematic groups, presented in a demonstration table. The Psychiatric Reform and Education in Mental Health are the main lines of scientific production. The ideological and social situation of each year related to scientific production, indicating that academy and assistance go together.
\end{abstract}

KEYWORDS: Mental health; Psychiatric nursing, Research.

\section{TENDENCIAS DE LAS INVESTIGACIONES DE ENFERMERÍA PSIQUIÁTRICA Y SALUD MENTAL EN PERIODO DE 1998 A 2008}

RESUMEN: El objetivo fue analizar la producción científica en los Encuentros de Investigadores en Salud Mental y Encuentro de Especialistas en Enfermería Psiquiátrica, en relación a los cambios ocurridos y a las políticas de Salud Mental en país, en periodo de 1998 a 2008. Los resúmenes fueron categorizados en 11 núcleos temáticos, presentados en una tabla demostrativa, siendo la Reforma Psiquiátrica y la Enseñanza en Salud Mental los principales ejes de producción científica. La situación ideológica y social de cada año se relaciona a las producciones científicas, indicando que el medio académico y la realidad asistencial caminan conjuntamente.

PALABRAS CLAVE: Salud mental; Enfermería psiquiátrica; Investigación.

${ }^{1}$ Acadêmica da Escola de Enfermagem da Universidade Federal de Minas Gerais-EE-UFMG.

${ }^{2}$ Enfermeira. Doutora em Enfermagem Psiquiátrica. Professor Adjunto do Departamento de Enfermagem Aplicada da EE-UFMG.

Autor correspondente:

Amanda Márcia dos Santos Reinaldo

Universidade Federal de Minas Gerais

Av. Alfredo Balena, 190 - 30130-100 - Belo Horizonte-MG, Brasil

Recebido: 12/02/10

E-mail: amsreinaldo@enf.ufmg.br

Aprovado: 07/07/10

Cogitare Enferm. 2010 Jul/Set; 15(3):528-35 


\section{INTRODUÇÃO}

O ensino da Enfermagem, historicamente, fundamentou-se na assistência, no saber prático, na maneira mais adequada de executar um determinado procedimento; por conseguinte, os profissionais da área voltam-se, em essência, à formação técnica, ignorando, em alguns momentos, a essência críticoreflexiva apresentada pela Enfermagem enquanto ciência.

Contudo, um crescente contingente de enfermeiros suscitou questionamentos referentes a essa prática desvinculada do saber científico, emergindo, portanto, a necessidade de alterar a formação então vigente, assim como o nível de escolaridade dos profissionais da área. Dessa forma, em meados da década de 40, nos Estados Unidos, principiaram-se os primeiros cursos de pós-graduação em Enfermagem, fazendo com que os enfermeiros brasileiros, principalmente aqueles envolvidos com a prática docente, ingressassem no processo de aprimoramento técnico-científico ${ }^{(1)}$.

Esses docentes brasileiros foram os primeiros orientadores de pesquisas e, em decorrência da criação dos cursos de pós-graduação no Brasil, na década de 70, foram, também, os principais incentivadores da pesquisa em Enfermagem. Sendo assim, a oferta dos referidos cursos atuou, de forma significativa, no desenvolvimento do saber científico dos enfermeiros da época, uma vez que a pesquisa representava um dos requisitos para a titulação dos mesmos, ratificando a importância da pós-graduação no fomento da pesquisa na Enfermagem ${ }^{(1)}$.

A Escola de Enfermagem de Ribeirão Preto (EERP), fundada em 1951, insere-se no processo de desenvolvimento, não unicamente da pesquisa em Enfermagem, mas também da própria profissão, atuando na graduação, na pós-graduação e no aperfeiçoamento científico de recursos humanos.

A educação continuada iniciou-se na EERP em 1975, no Departamento de Enfermagem Psiquiátrica e Ciências Humanas, com o Mestrado em Enfermagem Psiquiátrica - o terceiro no âmbito nacional. Esse curso configurou-se de suma relevância para o desenvolvimento da Enfermagem Psiquiátrica e Saúde Mental no país, ao proporcionar subsídios teóricopráticos, tanto nas instituições asilares quanto nos espaços preconizados pela Reforma Psiquiátrica ${ }^{(2)}$.

Dessa forma, a Reforma Psiquiátrica interferiu diretamente no ensino de Enfermagem Psiquiátrica e Saúde Mental, ao inserir os discentes nos serviços substitutivos, ou seja, em cenários práticos que primam por uma assistência humanizada, reintegradora e relacional, contrária ao reducionismo biológico ${ }^{(3)}$.

Além de ofertar cursos de pós-graduação, a Escola de Enfermagem de Ribeirão Preto contribui para o ensino e a pesquisa em Enfermagem Psiquiátrica, ao promover, a cada dois anos, o Encontro de Pesquisadores em Saúde Mental (EPSM), em conjunto com o Encontro de Especialistas em Enfermagem Psiquiátrica (EEEP). Juntos, eles reúnem acadêmicos, docentes e profissionais brasileiros e procedentes de outras nacionalidades interessados na temática.

Assim, o objetivo deste trabalho é analisar a produção científica nos EPSM e EEEP, em relação às mudanças ocorridas e às políticas de Saúde Mental no país, no período de 1998 a 2008.

\section{MÉTODO}

Trata-se de uma revisão de literatura na qual foram utilizados os Anais dos seis EPSM e EEEP, compreendidos no período de 1998 a 2008, produzidos a partir da temática contemplada no evento, sendo compostos pelos resumos apresentados, o ano e a programação. Sendo assim, essa revisão de literatura permite observar, compilar e compreender a situação e a evolução dos estudos e das pesquisas sobre Enfermagem Psiquiátrica e Saúde Mental no país.

A revisão de literatura é uma metodologia que vai ao encontro do objetivo proposto neste estudo, ao posicionar o leitor do estudo e o próprio pesquisador acerca dos avanços, retrocessos, ou áreas envoltas em polêmicas. Ela também fornece informações para contextualizar a extensão e a significância do problema que se maneja, portanto, tem função histórica e de atualização ${ }^{(4)}$.

Para tanto, realizou-se uma leitura, a princípio preliminar e, posteriormente, mais aprofundada, tanto dos resumos apresentados, quanto de material científico publicado em periódicos nacionais, documentos, leis e relatórios pertinentes ao tema, relacionando esse referencial com os trabalhos apresentados em cada volume dos Anais. Assim sendo, a partir das temáticas de cada encontro e da recorrência dos temas apresentados no mesmo, buscaram-se referências bibliográficas que contemplassem os conteúdos mais frequentes, como meio de fundamentar e justificar as tendências temáticas verificadas em cada recorte temporal. Após esse levantamento, procurou-se discutir os temas abordados a cada ano do evento na relação com o momento 
histórico-político da Saúde Mental no país.

Os 952 resumos, referentes aos Anais dos seis encontros, foram analisados e agrupados em 11 núcleos temáticos, conforme enfoque dado aos trabalhos e ano de apresentação no evento. Para melhor organização e visualização dos resultados, optou-se por demonstrá-los em forma de tabela, a qual contém o núcleo temático e o enfoque geral dos resumos nesse núcleo, além dos anos dos eventos, para que seja indicado o número de trabalhos de determinado núcleo em cada ano. A discussão dos resultados foi feita por ano, sendo debatidos, em especial, os núcleos temáticos com maior publicação de resumos.

\section{RESULTADOS E DISCUSSÃO}

No período compreendido entre 1998 e 2008, realizaram-se 06 EPSM e EEEP, cujos núcleos temáticos, com os respectivos enfoques gerais, apresentamse na tabela 1 .

Tabela 1 - Distribuição temática dos resumos apresentados no EPSM e no EEEP, 1998-2008.

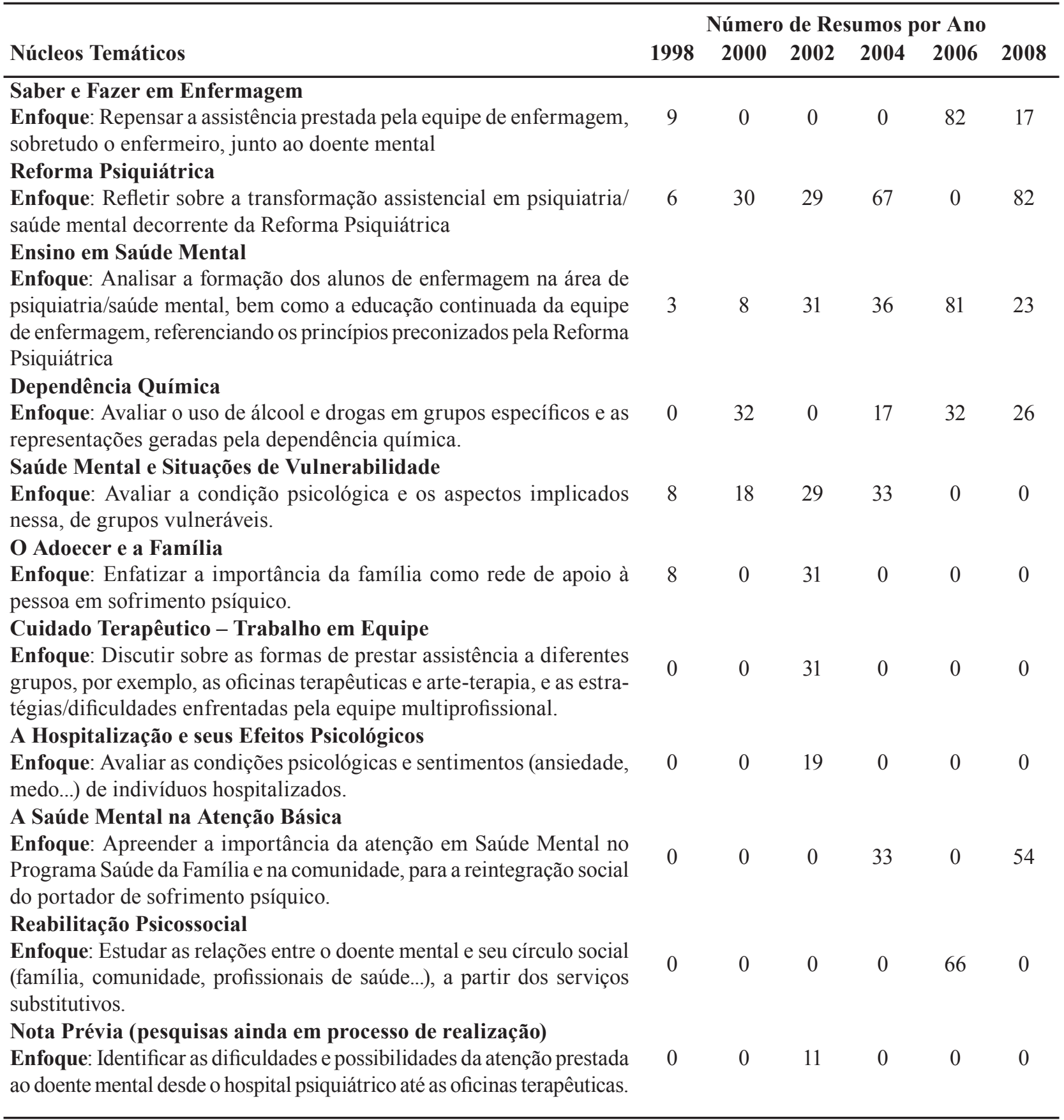


No ano de 1998, no período de 27 a 30 de abril, ocorreu o EPSM e o IV EEEP, cujo tema foi: Caminhando para a assistência integral, com 34 trabalhos publicados. Partindo-se do Caderno de publicações desse Encontro $^{(5)}$, evidenciou-se que o enfoque dos resumos reside na importância em se pensar o cuidado desenvolvido junto ao portador de sofrimento psíquico e à sua família de modo integral, rejeitando a assistência centrada unicamente na patologia, dissociada dos aspectos sociais e emocionais do paciente.

Essa nova visão ampliada referente ao portador de transtorno mental somente se tornou possível após a Reforma Psiquiátrica, que aponta a necessidade de acabar, de forma progressiva, com os manicômios, devolvendo ao doente mental sua liberdade e cidadania, porém sem deixá-lo desamparado no âmbito assistencial, dada a criação dos serviços substitutivos de atenção.

A década de 90 caracteriza-se pelo movimento de mudança na assistência prestada ao portador de transtorno mental, assim como na alteração do modelo de atenção psiquiátrica, que passou de hospitalocêntrico para os serviços substitutivos. Assim, nesse período, surgiram os primeiros serviços ambulatoriais de saúde mental, os Centros de Atenção Psicossocial (CAPS), os Centros de Convivência, dentre outros, buscando apontar novas direções de atendimento ao paciente, revertendo a lógica de segregação e discriminação dos hospitais psiquiátricos. Nessa década os objetivos estão mais concentrados na operacionalização de um novo modelo de assistência, bem como na realização de uma experiência territorializada, participativa, integral e interdisciplinar ${ }^{(6)}$.

Inseridos na comunidade, os serviços substitutivos devem primar por ações destinadas à família dos portadores de sofrimento mental, visando à potencialização da relação família-profissional-serviço. Mostra-se de suma relevância a inclusão do familiar na rede de cuidados, apreendendo também a sua demanda, a qual supera a objetividade expressa em suas queixas, atingindo seu próprio sofrimento psíquico ${ }^{(7)}$.

O contexto sociopolítico da década de 90 , certamente, relaciona-se ao enfoque dos trabalhos apresentados no Encontro de 1998, uma vez que existe relação e proximidade entre o contexto da época e os assuntos dos trabalhos.

Percebe-se que, após o ano de 1998, somente no ano de 2006 houve expressividade de resumos referentes ao Saber e Fazer em Enfermagem, voltada não mais aos Serviços de Atenção em Saúde Mental, como em 1998. Entende-se que isso se deve ao fato de que houve uma evolução significativa da Reforma Psiquiátrica no território brasileiro, levando à incorporação de nova temática nas disciplinas e nos currículos de formação de recursos humanos em Enfermagem.

Seguindo a ordem dos Encontros da EERP, em 2000, com os 88 trabalhos publicados, realizou-se o VI EPSM e V EEEP, cujo tema foi: O Uso e Abuso de Álcool e Drogas: Um Desafio para Todos ${ }^{(8)}$.

Nesse Encontro, percebe-se, em convergência com o tema proposto, uma predominância de resumos relacionados ao uso e abuso de álcool e drogas, em especial, referentes à importância de atividades educativas e preventivas sobre a dependência química. Essa temática, extremamente presente e crescente na sociedade brasileira, ainda se configura em um desafio social e assistencial, sendo de extrema importância desenvolver atividades informativas, de educação e prevenção, principalmente, entre jovens e adolescentes.

Em junho de 1998, o governo brasileiro criou o Sistema Nacional Antidrogas (SISNAD), composto pela Secretaria Nacional Antidrogas (SENAD) e pelo Conselho Nacional Antidrogas (CONAD). Esses novos órgãos federais apresentam, como objetivo, o enfrentamento do problema mundial das drogas, seja por meio de atividades de repressão ou de atividades de prevenção, quais sejam, divulgação de informação e educação social, dentre outras ${ }^{(9)}$.

Ainda em 1998, ocorreu o $1^{\circ}$ Fórum Nacional Antidrogas, que contou com a participação de diferentes grupos sociais vinculados ao tema, promovendo debates e discussões acerca do dependente químico, da forma de tratamento, da reinserção social, dentre outras temáticas, a partir dos quais se criou a Política Nacional Antidrogas (PNAD) ${ }^{(9)}$.

Além disso, verificou-se que, em meados do ano 2000, segundo relatórios de organizações internacionais, aproximadamente 200 milhões de pessoas consumiam algum tipo de droga ilícita ${ }^{(9)}$. Em meio a esse contexto social, é de suma importância que o meio científico também se mobilize na tentativa de diminuir os referidos índices, por meio de estudos, pesquisas e eventos que coloquem esse tema em destaque, como realizado no Encontro de 2000.

No ano de 2000, houve também um expressivo número de trabalhos no núcleo temático Reforma Psiquiátrica. Entretanto, no presente optou-se por abordar esse núcleo e discutir a temática em questão, de modo aprofundado, no ano de 2004, pela superioridade de trabalhos acerca da Reforma Psiquiátrica 
apresentados nesse ano.

Em 2002, realizou-se o VII EPSM e o VI EEEP, cujo tema foi: Cuidar: tão longe... tão perto... . Nesse ano, denotou-se o aumento no número de trabalhos inscritos, perfazendo um total de $181^{(10)}$.

A partir do núcleo de resumos de 2002, percebe-se uma ampla diversidade de assuntos que podem ser englobados pela Saúde Mental e Enfermagem Psiquiátrica, o que indica o amplo espectro de atuação dos profissionais e serviços da área. Além disso, os temas nos quais a publicação apresentou-se mais expressiva transparecem a dificuldade da equipe e da família em lidar com o paciente psiquiátrico, uma vez que os serviços substitutivos ainda apresentam limitações para sua implantação, o que deixa a assistência ora distante de alcançar os propósitos da Reforma Psiquiátrica, ora muito perto.

Ao realizar um retrocesso político-assistencial, no final da década de 1990, constatou-se que a redução do número de leitos em hospital psiquiátrico era acompanhada por uma criação insuficiente de serviços substitutivos em Saúde Mental, e que os poucos serviços não estavam aptos a atender a demanda de pacientes desospitalizados ou não funcionavam de acordo com o ideário da Reforma ${ }^{(6)}$.

Portanto, na tentativa de acelerar o processo de desinstitucionalização e de reinserção social, Paulo Delgado formulou a Lei n. 10.216, aprovada em 2001, com o intuito de garantir legalmente o direito dos portadores de sofrimento psíquico. Essa mesma Lei incentiva a humanização do tratamento do doente mental, vetando a internação do mesmo em instituições de caráter asilar, com algumas ressalvas, e estimulando a participação da família e da comunidade nesse processo de reintegração social ${ }^{(11)}$.

Em consonância com a Lei 10.216, o Ministério da Saúde criou a Portaria n ${ }^{\circ} 106$, em fevereiro de 2000, estimulando a criação dos serviços substitutivos em Saúde Mental, em especial, as Residências Terapêuticas, que também contribuem para a inserção social dos pacientes até então internados em instituições de longa permanência, sendo que, a cada transferência de paciente para essas moradias, o leito vago deve ser fechado ${ }^{(12)}$.

Ainda no Encontro de 2002, ao contrário dos demais, os resumos apontaram a relevância do trabalho em equipe pelos profissionais atuantes em saúde mental das diversas áreas, assim como os impasses e os entraves desencadeados por essa articulação de saberes.

Sendo assim, demandou-se de psiquiatras, psicólogos, enfermeiros e terapeutas ocupacionais, dentre outros, uma comunicação e uma prática integradas, respeitosas aos limites e às contribuições de cada categoria profissional, de modo a minimizar os conflitos e, principalmente, de proporcionar, ao portador de sofrimento psíquico, uma assistência mais humanizada e fundamentada nos aspectos biopsicosociais desses sujeitos ${ }^{(13)}$.

O núcleo temático $\mathrm{O}$ Adoecer e a Família também apresentou significativo número de trabalhos, o que indica a relevância da abordagem da família no acompanhamento e no tratamento do portador de transtorno mental.

Sabe-se que, não raro, a família constitui-se a primeira rede de contatos do portador de transtorno mental egresso de instituições manicomiais, apresentando, comumente, dúvidas e anseios acerca do seu papel no cuidado e na reabilitação psicossocial do portador de transtorno mental. Portanto, torna-se fundamental tanto a explicação e a valorização da mesma como agente ativo no projeto terapêutico do familiar portador de sofrimento psíquico, bem como na oferta de suporte, para que haja uma melhora na qualidade de vida do cuidador e de quem é cuidado ${ }^{(7)}$.

Ao analisar os resumos do ano de 2002 percebe-se um quantitativo grande de trabalhos no núcleo Ensino em Saúde Mental, porém como houve um número ainda mais expressivo de resumos dessa categoria no ano de 2006, optou-se por discutir tal temática nesse ano. Cumpre ressaltar, ainda, que o ano de 2002 constituiu-se o único com resumos no núcleo A Hospitalização e seus efeitos psicológicos.

Dando continuidade às edições dos Encontros, em 2004, a EERP realizou o VIII EPSM e o VII EEEP, sendo o tema norteador a Saúde Mental numa Sociedade em Mudança, com 186 trabalhos $^{(14)}$.

Os trabalhos apresentados nesse ano vinculam-se à mudança na assistência psiquiátrica e em saúde mental no Brasil, decorrente da Reforma Psiquiátrica, com enfoque expressivo na atenção básica e nos CAPS, em detrimento à estrutura manicomial.

Assim, nesse processo de inversão do modelo assistencial em saúde mental, insere-se o CAPS, configurando-se como um dispositivo central, o eixo articulador dos demais serviços substitutivos em saúde mental, que promove atendimento clínico em regime de atenção diária, o que evita a necessidade de internação psiquiátrica dos pacientes ${ }^{(15)}$.

O Ministério da Saúde aponta que a melhoria da assistência ao doente mental deve estar diretamente 
vinculada à atenção básica, para que os casos menos complexos possam ser atendidos junto à própria comunidade, sendo que o CAPS da região deve prestar apoio às equipes do Programa de Saúde da Família (PSF). Além disso, devido à proximidade com a família e a comunidade, a atenção básica constitui-se em um recurso estratégico para a saúde mental, em especial nos pequenos municípios onde não há necessidade de implantação de CAPS ${ }^{(15)}$.

Entretanto, inserir, de fato, a saúde mental na atenção básica constitui-se um dos principais desafios ao avanço da Reforma Psiquiátrica e para os profissionais que atuam na atenção primária. Percebe-se um despreparo e desinteresse de tais profissionais em acolher o portador de sofrimento psíquico; a ausência de interlocução intersetorial, por exemplo, com o CAPS de referência; uma resistência em desenvolver oficinas e grupos em saúde mental, com receio do aumento da demanda ${ }^{(16)}$.

De acordo com o exposto, e com os dados compilados, ressalta-se o ano de 2004 como um marco no desenvolvimento assistencial substitutivo, no qual ocorreu expansão significativa dos CAPS devido ao financiamento do Ministério da Saúde, e, com o crescente desenvolvimento do Programa Saúde da Família no país, explica-se a predominância de trabalhos englobando esses assuntos ${ }^{(16)}$.

No ano de 2006, o IX EPSM e VIII EEEP propuseram o tema Os novos velhos desafios da saúde mental e, nesse Encontro, publicaram-se 261 traba1 hos ${ }^{(17)}$. Partindo-se da análise da tabela demonstrativa, percebe-se um predomínio de trabalhos relacionados ao ensino em Enfermagem Psiquiátrica/Saúde Mental, bem como, ao saber e fazer em Enfermagem, núcleos temáticos esses que se inter-relacionam no cenário assistencial.

Em paralelo às mudanças na assistência desencadeadas pela Reforma Psiquiátrica, denotou-se a necessidade de o ensino adequar-se a esse novo modelo, alterando a ênfase das disciplinas de Enfermagem Psiquiátrica e Saúde Mental. Reduziram-se, dos programas curriculares, as abordagens psicopatológicas, atribuindo equânime relevância aos aspectos sociais e emocionais do portador de transtorno mental. Dessa forma, novos modelos de cuidado inserem-se no cronograma das disciplinas, como, por exemplo, o relacionamento e a comunicação terapêuticos, evidenciados, essencialmente, no contexto pós-Reforma ${ }^{(3)}$.

Igualmente, mostra-se de fundamental importância que o aluno experimente a prática, conheça as ações de enfermagem para com o doente mental, o manejo clínico com paciente em sofrimento psíquico, para que o discente possa conhecer o outro, e a si mesmo, por meio da ruptura de seus medos, ansiedades e, talvez, preconceitos ${ }^{(3)}$.

Nesse contexto, insere-se a íntima relação entre o ensino de Saúde Mental e o saber-fazer em Enfermagem Psiquiátrica, uma vez que as concepções desenvolvidas pelos trabalhadores inseridos nos diversos serviços de saúde mental, acerca do objeto e da finalidade do trabalho, moldam-se em consonância com o estabelecimento assistencial.

Portanto, o ensino clínico em saúde mental constitui-se em um momento em que o discente depara-se com as concepções institucionalmente propaladas, aproximando sua formação, e futura atuação profissional, às vertentes que sustentam o espaço de estágio. Assim sendo, o ensino representa o saber-fazer em enfermagem psiquiátrica futuro, desvelando a relevância de o ensino e a assistência em saúde mental caminharem paralelamente, seguindo princípios em comum, com por exemplo a reabilitação psicossocial do portador de transtorno mental.

A reabilitação psicossocial, um dos núcleos temáticos expressivos do ano de 2006, calca-se na reinserção social do sujeito em sofrimento psíquico, de modo que seus laços familiares, afetivos e contratuais possam ser restabelecidos paulatinamente. Dessa forma, esse sujeito reassume o seu papel social, como um ser autônomo e dotado de poder decisório, ao contrário do histórico cunho biológico e reducionista com que se tratava o paciente psiquiátrico.

Concluindo a análise dos Cadernos, em 2008, no X EPSM e no IX EEEP - I Simpósio LatinoAmericano de Saúde Mental, com a temática A Saúde Mental em Tempos de Reformas Curriculares" - foram apresentados 202 trabalhos $^{(18)}$.

A partir dos núcleos temáticos presentes no Encontro de 2008, percebe-se uma supremacia de trabalhos vinculados à Reforma Psiquiátrica e à assistência psiquiátrica na Atenção Básica.

Os princípios preconizados pela Reforma Psiquiátrica voltam-se a uma redução do artificial distanciamento que se firma entre a pessoa em sofrimento, o profissional de saúde, os familiares e a comunidade em geral. Assim sendo, a atenção básica, inserida, espacial e simbolicamente, na comunidade permite aproximar o portador de transtorno mental aos espaços sociais que perfazem o seu ciclo vital.

Para tanto, a escuta qualificada constitui-se 
em uma estratégia incomensurável no delineamento do itinerário terapêutico e social do doente mental, possibilitando a valorização do seu discurso, não somente em relação ao seu sofrimento, mas, igualmente, ao serviço e ao espaço comunitário ao qual o mesmo pertence. Contemplar a essência discursiva do usuário permite a humanização do cuidado, a compreensão de suas demandas físico-sociais e, por conseguinte, a construção dos passos futuros de seu plano terapêutico.

Sendo assim, partindo-se do tema proposto nesse Encontro, denota-se a necessidade de integrar, aos currículos das instituições formadoras de todo o país, disciplinas/conteúdos que enfatizem as competências relacionais e a integralidade do cuidado à pessoa em sofrimento psíquico.

\section{CONSIDERAÇÕES FINAIS}

Partindo-se da análise dos Anais desse evento, percebe-se o estímulo e a contribuição para o desenvolvimento e a divulgação de trabalhos dessa área, por meio de discussões e mesas-redondas cujos temas incitam o desenvolvimento de um pensamento crítico e, consequentemente, geram curiosidade epistemológica nos discentes e docentes de diversas regiões do país.

A realização desse evento, além de proporcionar o crescimento de publicações científicas, conforme evidenciado na evolução do contingente de trabalhos apresentados no decorrer dos Encontros estudados, confronta as diversas realidades sociais e de formação de recursos humanos existentes no país, possibilitando um intercâmbio de experiências positivas e negativas, o que se reflete diretamente na assistência prestada ao portador de transtorno mental.

A categorização e a análise do material científico produzido nos Encontros possibilitam a apresentação e a articulação, concretas, entre o meio acadêmico, as Políticas de Saúde Mental e a prática profissional desenvolvida nessa área, apontando temáticas recorrentes e que demandam maior atenção, tanto dos interessados em Saúde Mental, quanto da escolha da temática de eventos futuros.

Além disso, os trabalhos apresentados apontam uma relação explícita com as mudanças legislativas, sociais e ideológicas, enfim, com a política governamental de cuidado ao portador de sofrimento psíquico. Sendo assim, esse evento de caráter científico apresenta-se consciente e vinculado ao contexto e às tendências sociopolíticas em Saúde Mental no Brasil, ao propor temas consoantes à conjuntura social de cada época.
Dessa forma, percebe-se que o contexto sociopolítico apresenta uma influência direta nas inquietações e nas produções científicas dos enfermeiros dessa área, apontando que o meio acadêmico e a realidade social estão muito mais interligados do que se poderia supor inicialmente.

Sendo assim, esse estudo possibilita um acompanhamento da prática desenvolvida em Enfermagem Psiquiátrica e Saúde Mental, indicando os principais avanços e entraves na assistência ao portador de transtorno mental. Assim, acarreta em uma reflexão crítica acerca das possibilidades e potencialidades do cuidado em saúde mental, refletindo, diretamente, na atenção dispensada às pessoas em sofrimento psíquico.

\section{REFERÊNCIAS}

1. Santos TCF, Gomes MLB. Nexos entre pós-graduação e pesquisa em Enfermagem no Brasil. Rev Bras Enferm. [Internet] 2007;60(1) [acesso em 15 jan 2010]. Disponível: http://tiny.cc/sw3ua

2. Furegato ARF, Saeki T. Formando especialistas em enfermagem psiquiátrica e saúde mental por mais de duas décadas. In: Compreensão e crítica para uma clínica de enfermagem psiquiátrica. Cad IPUB, 2000;7(19):128-41.

3. Kantorski LP, Pinho LB, Saeki T, Souza MCBM. Relacionamento terapêutico e ensino de enfermagem psiquiátrica e saúde mental: tendências no estado de São Paulo. Rev Esc Enferm USP. [Internet] 2005;39(3) [acesso em 17 jan 2010]. Disponível: http://tiny. $\mathrm{cc} / 4 \mathrm{w} 6 \mathrm{q} 3$

4. Noronha DP, Ferreira SMSP. Revisões de literatura. In: Campelo BS. Condón BV, Kremer, JM, organizadores. Fontes de informação para pesquisadores e profissionais. Belo Horizonte:UFMG, 2000

5. Anais do V Encontro de Pesquisadores em Saúde Mental, IV Encontro de Especialistas em Enfermagem Psiquiátrica. Caminhando para a assistência integral; 1998 abr 27-30; Ribeirão Preto. São Paulo: Departamento de Enfermagem Psiquiátrica e Ciências Humanas da EERP-USP; 1998.

6. Nunes M, Torrente M, Ottoni V, Moraes Neto V, Santana M. A dinâmica do cuidado em saúde mental: signos, significados e práticas de profissionais em Centro de Assistência Psicossocial em Salvador, Bahia, Brasil. Cad Saúde Pública. [Internet] 2008;24(1). [acesso em 06 jan 2010]. Disponível: http://tiny.cc/tot7j 
7. Pereira MAO, Pereira Junior A. Transtorno mental: dificuldades enfrentadas pela família. Rev Esc Enferm USP. [internet] 2003;37(4) [acesso em 16 jan 2010]. Disponível: http://tiny.cc/cb0az

8. Anais do VI Encontro de Pesquisadores em Saúde Mental, V Encontro de Especialistas em Enfermagem Psiquiátrica. O Uso e Abuso de Álcool e Drogas: Um Desafio para Todos; 2000 abr 17-20; Ribeirão Preto. São Paulo: Departamento de Enfermagem Psiquiátrica e Ciências Humanas da EERP-USP; 2000.

9. Machado AR, Miranda PSC. Fragmentos da história da atenção à saúde para usuários de álcool e outras drogas no Brasil: da Justiça à Saúde Pública. Hist. Cienc Saúde Manguinhos. [Internet] 2007;14(3) [acesso em 18 jan 2010]. Disponível: http://tiny.cc/m0g2g

10. Anais do VII Encontro de Pesquisadores em Saúde Mental, VI Encontro de Especialistas em Enfermagem Psiquiátrica. Cuidar: tão longe... tão perto...; 2002 mar 25-28; Ribeirão Preto. São Paulo: Departamento de Enfermagem Psiquiátrica e Ciências Humanas da EERP-USP; 2002.

11. Brasil. Lei n. 10216, de 6 de abril de 2001. Dispõe sobre a proteção e os direitos das pessoas portadoras de transtornos mentais e redireciona o modelo assistencial em saúde mental. Diário Oficial da União, [Internet] 7 Abr 2001 [acesso em 16 jan 2010]. Disponível: http:// tiny.cc/umhnu

12. Ministério da Saúde (BR). Portaria n. 106/GM, de 11 de Fevereiro de 2000. Institui os Serviços Residenciais Terapêuticos. Diário Oficial da União, [Internet] 12 Fev 2000 [acesso em 18 jan 2010] Disponível: http:// tiny.cc/r2lio

13. Büchelle F, Laurindo DLP, Borges VF, Coelho EBS. Interface da saúde mental na atenção básica. Cogitare Enferm. [Internet] 2006;11(3) [acesso em 18 jan 2010]. Disponível: http://tiny.cc/2mnll

14. Anais do VIII Encontro de Pesquisadores em Saúde Mental, VII Encontro de Especialistas em Enfermagem Psiquiátrica. Saúde Mental numa Sociedade em Mudança; 2004 abr 5-8; Ribeirão Preto. São Paulo: Departamento de Enfermagem Psiquiátrica e Ciências Humanas da EERP-USP; 2004.

15. Ministério da Saúde (BR). Conferência Regional de Reforma dos Serviços de Saúde Mental: 15 anos depois de Caracas. Reforma Psiquiátrica e política de saúde mental no Brasil. Brasília; 2005.
16. Brêda MZ, Silva Augusto LG. O cuidado ao portador de transtorno psíquico na atenção básica de saúde. Cienc \& Saúde Colet. [Internet] 2001;6(2) [acesso em 18 jan 2010]. Disponível: http://tiny.cc/pchxs

17. Anais do IX Encontro de Pesquisadores em Saúde Mental, VIII Encontro de Especialistas em Enfermagem Psiquiátrica. Os novos velhos desafios da saúde mental; 2006 mar 27-30; Ribeirão Preto. São Paulo: Departamento de Enfermagem Psiquiátrica e Ciências Humanas da EERP-USP; 2006.

18. Anais do X Encontro de Pesquisadores em Saúde Mental, IX Encontro de Especialistas em Enfermagem Psiquiátrica - I Simpósio Latino-Americano de Saúde Mental. A Saúde Mental em Tempos de Reformas Curriculares; 2008 jun 9-12; Ribeirão Preto. São Paulo: Departamento de Enfermagem Psiquiátrica e Ciências Humanas da EERP-USP; 2008. 